\title{
Knowledge Management by Reusing Experience
}

\author{
Sabine Delaître ${ }^{1}$ and Sabine Moisan ${ }^{2}$. \\ ${ }^{1}$ ENSMP, Pôle Cindyniques, BP 207, F-06904 Sophia Antipolis, France \\ Sabine.Delaitre@cindy.cma.fr \\ ${ }^{2}$ INRIA, projet Orion, BP 93, F-06902 Sophia Antipolis, France \\ Sabine.Moisanesophia.inria.fr
}

\begin{abstract}
The paper presents a study of the use of past experience in emergency management with an application to Forest Fire-Fighting Management. Our objective is the sharing of experience between the emergency managers in order to support the re-planning task, regarded as the relevant task of Forest Fire-Fighting Management. Therefore it is necessary to establish a common working language to facilitate sharing. First, we present the methodology used to determine the key element of this language, which is called a unit of experience. We then introduce a method to capitalize such units. Next, we describe the utilization of units of experience to improve emergency management. The reasoning method provides several levels of support to fire managers.
\end{abstract}

\section{Introduction}

This paper studies how reasoning from experience can improve risk management. Risk can be managed by means of different missions [1]. Among them, we are mostly interested in training and in intervention. This paper focuses on intervention like in [2],[3] but by reusing the experience itself which is regarded as a new way to assist emergency management [4]. We put our knowledge management approach into practice in Spain within the Andalusian organization of Forest Fire-Fighting Management (FFFM). The objective is to realize an experience-based support system which allows the fire managers to share their past experience. Among the managers' tasks, we decided to focus on the task of revising a fighting plan, or in others words on replanning because it presented the opportunity to exploit past experience. We believe that it is the best choice for validating the contribution of the sharing of experience both in intervention and training. FFFM implies taking into account two essential aspects of forest fire-fighting: the phenomena which govern the fire propagation and the intervention (effect of fighting resources, procedures applied for prevention and fighting, etc.). Interactions between these aspects generate the complexity of FFFM. This complexity must be considered during the constitution of the corporate memory that will be used for sharing and reusing knowledge. The problem is how to capture this complexity without it being detrimental to sharing and reusing of the experience, that we want to favor. 
Our approach consists of a formalization of the related experience and its capitalization in a corporate memory. Contrary to other knowledge management approaches, we do not store "compiled" experience (e.g., in the form of statistics) but the experience "as is". In our approach the concrete experience from past fire-fighting will be reused, without transformation. Moreover, in order to allow an efficient and sustainable sharing of experience, we propose structuring the experience, to constitute a common working language among fire managers.

The paper first introduces the key element of this language, which is named a unit of experience. The second part presents the method to capitalize such units and deals with the different types of decision support which can be provided to forest fire managers. Finally, we describe the construction of a unit of experience and we compare our approach to others in the domain of knowledge management.

\section{Unit of Experience}

We propose to model the experience of fire managers by a set of so-called units of experience, that will constitute the basic elements of the corporate memory about Forest Fire-Fighting Management. The formalization of such units takes into account the previous aspects of forest fire-fighting. Each unit of experience describes a resolved episode of the revision of a fighting plan. We have determined that this is the smallest element of the fire-fighting that preserves the complexity of the re-planning task, while providing useful information for experience sharing. First, a study of the phenomenal aspect enables us to decompose the fire evolution and to define the scope of units of experience. Second, the study of the intervention allows us to identify information relevant to experience used during the re-planning task. We hence specified a structure for units of experience that is easy to understand by managers and easy to process by a system.

\subsection{Unit of Experience Scope}

In our case, the information on the phenomenal aspect corresponds to an account of fire-fighting given by a manager. FFFM is described by a temporal sequence of situations, each one being the representation of the state of fire-fighting at any one moment. The temporal sequencing of situations is determined by so-called disruptive events, which trigger the revision of a plan (e.g., an accident of a fighting resource). The revision starts when the event revokes the current plan, i.e. when it revokes at least one of its sub-plans, leading to a new fighting situation. A situation is in turn described by a plan composed of sub-plans. Each sub-plan is associated to one sector, i.e. a particular geographic zone, with a given vegetation, topography or infrastructures which necessitate a specific fighting sub-plan. A sub-plan is defined by a set of operations (e.g., "build a break-line") and resources (e.g., fire trucks) to achieve a fighting goal, local to a sector (e.g., "protect a house"). FFFM hence decomposes temporally into situations and spatially into sectors as shown in Figure 1. 


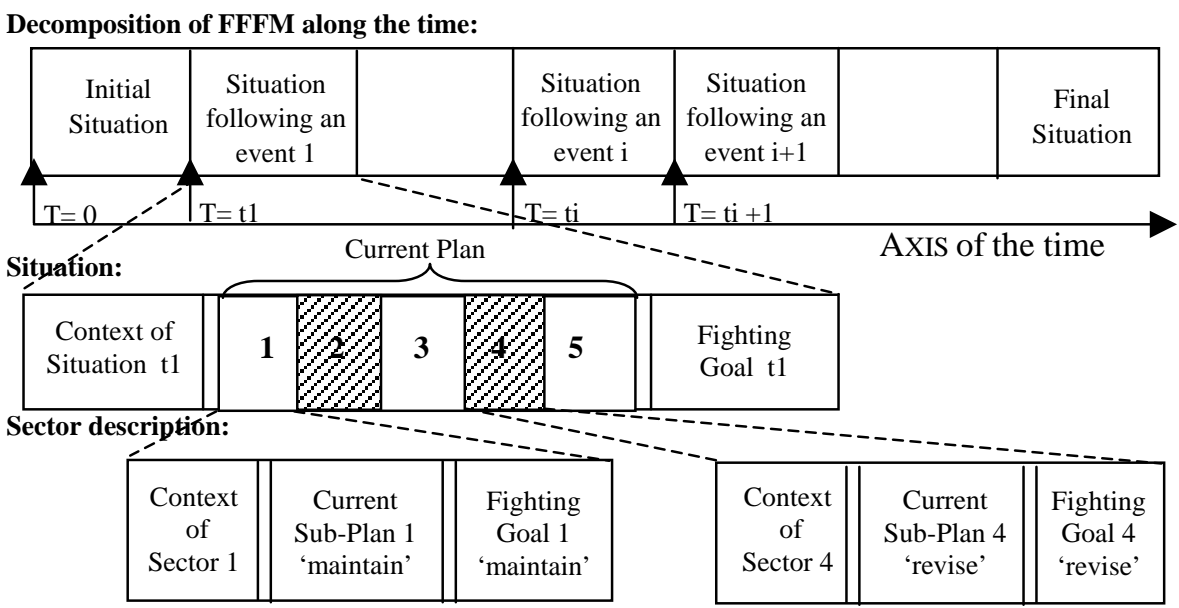

Fig. 1. Twofold decomposition of a Forest Fire-Fighting Management.

This analysis allows us to limit the scope of a unit to a sector of a given situation, where the sub-plan must be 'revised' in response to an event (like sector 4 in Fig.1).

\subsection{Unit of Experience: Relevant Information and Structure}

The knowledge that should be stored in a unit of experience corresponds to the knowledge relevant to the re-planning task of fire-fighting, where the sharing of experience occurs.

\begin{tabular}{|l|l|}
\hline \multicolumn{1}{|c|}{ UNIT OF EXPERIENCE STRUCTURE } & BUILDING STEP \\
\hline Problem description, i.e. & \\
\hline - Current plan context (Environmental context and fighting context), \\
- Description of the current fighting plan, \\
- Event that lead to a revision,
\end{tabular}

Remark: step 4 gives information about $\Delta$ plan in order to justify the actions and decisions taken.

Fig. 2. Structure of a unit of experience and identification of its building steps. 
First, the context of the current sub-plan is important to store. It consists of the environmental context (topography, vegetation, meteorology), and the fighting context (behavior of the fire, infrastructures and resources available to elaborate the modifications of the sub-plan). Furthermore, the modifications that have been applied and their effects are also stored. They represent the solution to the problem of how to revise a plan and the assessment about this solution. All this information is structured into operational units of experience which model episodes of revision of plans, like cases in case-based planning systems [5]. Their structure captures the previous information in a way that helps the storing and reusing of the experience. During the reasoning of the system the contents of the unit of experience is incremented in four steps (see Fig.2.) which are detailed in figure 4. Each unit of experience gathers all the information representing one cycle of the fire-fighting management (problem, solution, and outcome). That means that it cannot be divided into smaller parts, without losing the complexity of the FFFM otherwise it could not be used anymore for an experience-based support. The unit of experience is the smallest element that encapsulates the relevant experience, at the lowest possible level of complexity.

\subsection{Reusability of the approach}

Our approach can be adapted to other domains where emergency management applies. We describe a system (in a systemic approach) by a set of concepts: the phenomena, the behavior of phenomena, and the intervention. These concepts define an application ontology (described in [6] as a framework for both domain knowledge and reasoning method knowledge). A unit of experience describes an episode of plan revision. Therefore it contains information about the previous concepts and it constitutes the essential part of the problem description. This systemic view, and the derived unit of experience notion, are applicable to assist decisions during the replanning task of intervention in emergency management in general. The concepts of the ontology can be instantiated by domain specific terms. For the FFFM domain, the phenomena is the fire, i.e. the parameters which describe the fire (fire perimeter features, propagation, calorific power). The behavior of phenomena is the behavior of the fire i.e. the parameters which govern this behavior (vegetation, topography, meteorology) and the intervention is the fire-fighting i.e. the components of fighting plans.

\section{Management of Units of Experience}

We have designed an experience-based decision support system, the reasoning of which is decomposed into four phases : Initial capitalization, Elaboration of plan revision in response to an event, Resolution and Final capitalization. 


\subsection{Capitalization of Units of Experience}

The first and most important step is the collection of information. In Andalusia, the usual tool to collect information about fire development and management was a set of forms. But they did not contain all the relevant information for the needs of units of experience. This lead us to create new forms suitable to promote the sharing of experience for the revision of plans. The new forms fall into two types, corresponding to two types of knowledge acquisition [7]. The first type is filled out during the FFFM and captures the facts and the experience about the fire and the fighting development that are relevant for re-planning purposes. Thus we obtain a description of the concrete experience "as is"; this is the initial capitalization. The forms of second type are filled out after the FFFM and allow the managers to develop a critical point of view based on their experience (i.e. to justify their decisions or to propose alternatives). They provide understanding of the FFFM that has been made, i.e. they contain a valorization of the concrete experience. They correspond to the final capitalization. The fire management staff, using a real case of fire, validated the new forms.

\subsection{Reutilization of Units of Experience : Experience-Based Support}

The capitalized units of experience are used in a software system to provide support for FFFM. The different levels of proposed support correspond to the different steps of a re-planning task, as summarized in figure 3.

\begin{tabular}{|c|cc|c|}
\hline SUPPORT SYSTEM & \multicolumn{2}{|c|}{ SUPPORT LEVELS } & STEPS OF RE-PLANNING TASK \\
\hline Definition process (Elaboration) & 1. & perception support & perception of the problem \\
\hline Retrieval process (Resolution) & 2. & analysis support & identification of useful experience \\
\hline Adaptation process (Resolution) & 3. & action support & modifications of plan \\
\hline
\end{tabular}

Fig. 3. Levels of assistance to the re-planning task.

The two important phases, regarding the support levels, are the elaboration and resolution phases. The first phase splits up a problem into sub-problems in order to minimize the revision work. The second phase is divided into two processes (retrieval and adaptation) based on a CBP paradigm [8]; it solves each sub-problem, i.e. each 'revised' sub-plan (see Fig.1.). These phases constitute our cycle of plan revision whose specificity, compared to a classical CBP cycle, lies in the additional elaboration phase. This latter phase diagnoses the impact of an event, it corresponds to a dynamic definition process which uses inference rules, in order to define the signature of the problem (i.e. objective of the plan, relevant parameters and spot of adaptation). Thus, the first level of support is a perception support, which proposes explanations on how to interpret the occurring events according to the current fire-fighting plan. The retrieval process of the resolution phase aims to retrieve past units of experience. This process is driven by the objective of plan revision, used to apply a filter, and by the relevant parameters, used to compute a basic similarity measure (an Euclidean distance). After the retrieval process the user could obtain information about past fire-fighting management that has been selected as a useful experience to 
define a revision. This second level of support is named an analysis support. Then, the adaptation process works on past units in order to obtain solutions that may contribute to solving the current problem. The adaptation process, driven by the spot of adaptation, is applied to the retrieved solution plans. For this purpose, we use domain specific methods (following a transformational approach [9]), which are based on the elementary planning actions: insert, remove, reorder, etc. [10]. Some solutions could be suggested to the user. This third level hence proposes an action support. However, this last level of support is not always possible because the adaptation process requires a lot of knowledge, which is not always available. Among the support levels, only levels 2 and 3 use past experience, after respectively a selection and a modification. Figure 4 summarizes the support system and presents the way units of experience are built during and after FFFM. The retrieval process2 (see Fig.4.) is a simple request on a unit's identifier (fire and situation identification).

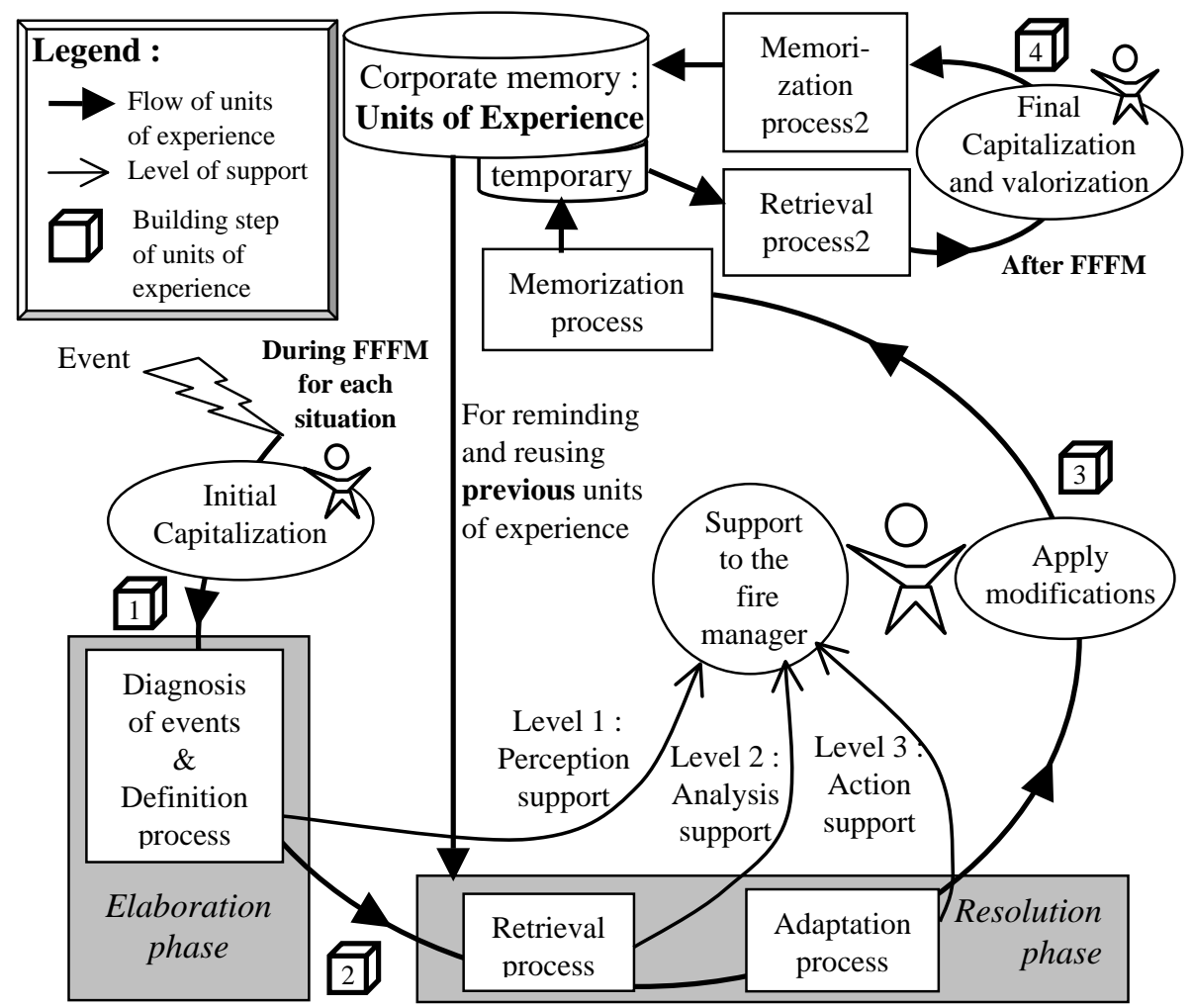

Fig. 4. Construction and reuse of the units of experience in the support system.

\subsection{Sharing of Units of Experience}

The first result of this study is to promote the collecting of information to acquire corporate experience. The model of unit of experience has been accepted by the man- 
agers and constitutes a common working language for the description of re-planning tasks. It offers an efficient way to exchange experiences and may constitute in the near future an opportunity to increase the corporate memory with alternate units of experience. One interesting aspect is the identification of "positive experience", which means the proposal of alternate solutions that could have improved this very same situation. These alternate solutions play the same role as the "missed opportunities" mentioned in [11]. The units stored in the corporate memory may also constitute a source of reference cases to favor training, either by working from a single unit to suggest alternate solutions, or from a series of units to build realistic scenarios.

\section{Conclusion and Perspectives}

Several Knowledge Management methods exist, that are not always supported by software tools, we can cite the MKSM method [12], which covers all aspects of knowledge management. Our objective is more focussed and aims to operationalize the capitalization and reuse of knowledge in the form of experience.

In order to improve emergency management by means of the sharing of experience between different managers, we have proposed the notion of unit of experience. Such a unit is not only a unit of memory, but also a unit of reasoning. The capitalization of units of experience leads to a corporate memory, regarded as an active organizational memory [13] and which constitutes the knowledge base of the reasoning process of our support system. This system follows a cycle close to the feedback life-cycle of experience promoted in the REX method [14]. REX is an experience management method that attempts to capture and reuse the experience. Compared to this method, we provide a second cycle for capitalization and valorization and we offer a computer assisted formalization of "elements of experience", that preserves the initial experience all around the cycle.

The need to learn lessons from past accidents, incidents, etc. is accepted by the risk management community [15],[16]. Debriefing methods promote post-operational evaluations to achieve this aim. In most cases, debriefing is used to define causes of events, but it has also more recently been used to study crisis management and we have adopted this new approach. But, instead of capitalizing only "negative facts", which is generally the case, we integrate and reuse both negative and positive past experience. Indeed, even if debriefing methods have influenced our knowledge management approach, we differ from them according to several points. First, we propose to capitalize not only the facts, but also the experience, and our approach is not only concerned with learning lessons from emergency management but also with understanding and explaining it, because the experience integrates criticisms from the managers themselves about the past fighting. The second point concerns memory organization. Our goal is to build a corporate memory containing the experience of different managers, and which is essential to achieving the sharing of experience. Finally, the third point is related to the sharing of knowledge. Knowledge corresponds to facts in debriefing approaches, while it corresponds to experience in ours. In debriefing methods, the sharing is not achieved because knowledge is not memorized during the 
debriefing phases. What is more, the analysis process gives as output a computation of facts (e.g., statistics). On the contrary, our approach preserves the experience from the capitalization phase until the sharing phase. The work presented in this paper lead to both an European project and the use of our forms by the training staff.

The presented unit of experience is sufficient to allow the reusing of concrete experience but there are some limits. That is why we intend to use another kind of unit representing schemas (as defined in [17]) or, in other words, plan hierarchies (as used in planning systems [18]). This second kind of unit will not represent an episode of the plan revision, but an abstracted and generalized experience.

\section{References}

1. Dykstra, D.P.:Systèmes d'information appliqués à la foresterie. Unasylva 189. Vol. 48, (1997), 10-15.

2. Xanthopoulos, G.: Development of a Decision Support System for water bomber dispatching in Greece. In Proc. $2^{\text {nd }}$ Int. Conf. FFR. Vol. I, A.08, Coimbra, (1994), 139-150.

3. Avesani, P., Perini, A. and Ricci, F.: Combining CBR and Constraint Reasoning in Planning Forest Fire Fighting. EWCBR'93. Springer-Verlag. (1993).

4. Huet, P.: Risques naturels et retour d'expérience: est-ce pour bientôt? Retours d'Expérience, Annales des Ponts et Chaussées. n91, Septembre, (1999), 54-61.

5. Muñoz-Avila, H., Hendler, J.A. and Aha, D.W.: Towards a Conversational Case-Based Planning Tool. (1998). Review of Applied Expert Systems.

6. Studer, R., Benjamins, R. and Fensel, D.: Knowledge Engineering: Principles and Methods. Data and Knowledge Engineering. 25(1-2), March, (1998).

7. Delaître, S., Mille, A. and Moisan, S.: Instrumentation d'un processus de retour d'expérience pour la gestion des risques. IC2000. May (2000).

8. Hammond, K.J.: CBP - Viewing planning as a memory task. Academic Press Inc. (1989).

9. Carbonell J.G.: Learning by Analogy: Formulating and Generalizing Plans from Past Experience. In Machine Learning: An Artificial Intelligence Approach. (1983), 137-161.

10. Hank, S. and Weld, D.S. : A Domain Independent Algorithm for plan-adaptation. Journal of Artificial Intelligence Research 2, (1995), 319-360.

11. Jarke, M.: Experience-Based Knowledge Management. In A.Solvberg, S.Brinkkemper, E. Lindencrona. (eds.): Information Systems Engineering, Springer-Verlag, June (2000).

12. Ermine, J-L.: Capitaliser et partager les connaissances avec la méthode MKSM. Traité IC2. Volume "Capitalisation des Connaissances », Hermès, (2000).

13. Sorli. A., Coll. G.J., Dehli. E., and Tangen. K.: Knowledge Sharing in Distributed Organisations. IJCAI-99 Workshop on KM and Organizational Memories. (1999).

14. Malvache, P., Eichenbaum, C. and Prieur, P.: La maîtrise du retour d'expérience avec la méthode REX. Performances Humaines et Techniques. n69, Mars-Avril (1994), 6-13.

15. Greenlee, J.: Possible Lesson Learned in Florida. Wildfire. Vol.7, ${ }^{\circ} 11$, (1998), 12-14.

16. Gilbert, C.: Premiers éléments de réflexion pour une approche transversale du retour d'expérience. Retours d'Expérience, Annales des Ponts et Chaussées. n91, (1999), 4-10.

17. Turner, R.M.: Adaptative Reasoning for Real-World Problems : A Schema Based Approach. LEA Publischers, Hillsdale, New Jersey, (1994).

18. Tate, A., Hendler, J. and Drummond, M.: A review of AI Planning Techniques. In Readings in Planning, J. Allen, J. Hendler, and A. Tate. (eds): Morgan Kaufmann Publishers, (1990), 26-49. 